\title{
Formation of terrestrial planets in close binary systems: The case of $\alpha$ Centauri A
}

\author{
M. Barbieri ${ }^{1}$, F. Marzari ${ }^{2}$, and H. Scholl ${ }^{3}$ \\ ${ }^{1}$ CISAS, c/o Dipartimento di Fisica, Università di Padova, via Marzolo 8, 35131 Padova, Italy \\ e-mail: mbarbier@pd.infn.it \\ 2 Dipartimento di Fisica, Università di Padova, via Marzolo 8, 35131 Padova, Italy \\ e-mail: marzari@pd.infn.it \\ 3 Observatoire de la Côte d'Azur, BP 4229, 06304 Nice Cedex 4, France \\ e-mail: scholl@obs-nice.fr
}

Received 3 April 2002 / Accepted 22 August 2002

\begin{abstract}
At present the possible existence of planets around the stars of a close binary system is still matter of debate. Can planetary bodies form in spite of the strong gravitational perturbations of the companion star? We study in this paper via numerical simulation the last stage of planetary formation, from embryos to terrestrial planets in the $\alpha$ Cen system, the prototype of close binary systems. We find that Earth class planets can grow around $\alpha$ Cen A on a time-scale of 50 Myr. In some of our numerical models the planets form directly in the habitable zone of the star in low eccentric orbits. In one simulation two of the final planets are in a 2:1 mean motion resonance that, however, becomes unstable after 200 Myr. During the formation process some planetary embryos fall into the stars possibly altering their metallicity.
\end{abstract}

Key words. planetary nebulae: general

\section{Introduction}

Planetary formation in close binary systems is still an open problem. Accretion disks have already been observed around each individual component (Rodriguez et al. 1998) strongly supporting the idea that at least the initial stage of planetary formation can occur. However, it is still uncertain whether the dust can coalesce into planetesimals and, in particular, whether the process of planetesimal accretion can continue till the completion of a planet. Marzari \& Scholl (2000) showed that relative velocities between small planetesimals are low in spite of the strong gravitational perturbations due to the companion star. The combined effects of gas drag and secular perturbations induce a sharp periastron alignment of the planetesimal orbits and, as a consequence, low relative speeds at impact that favor accumulation rather then fragmentation. However, the gravitational perturbations from the companion star may still halt the planetary formation process in the last stage when planetary embryos collide together to form a planet. In this paper we numerically model this final scenario where large embryos collide and, eventually, form larger bodies that can be termed planets.

We have concentrated on the Alpha Centauri system, the prototype of close binary systems with the more massive star very similar to our Sun. It is also the same system studied by Marzari \& Scholl (2000). Planetary embryos are likely to form

Send offprint requests to: M. Barbieri, e-mail: mbarbier@pd.infn.it within 2.5 $\mathrm{AU}$ of the star since beyond the companion perturbations are too strong and planetesimal collisions may lead preferentially to fragmentation rather then accretion (Marzari \& Scholl 2000). Moreover, orbits farther than 2.7 AU become unstable according to Holman \& Wiegert (1999). We model the accumulation of Lunar-size embryos into terrestrial planets to estimate the time-scale of formation and the dynamical and physical properties of the final planets. Different initial conditions are considered to have a statistical description of the possible final systems.

\section{The numerical model}

We have performed $26 \mathrm{~N}$-body simulations where we have numerically integrated the orbits of planetary embryos and of the companion star for $10^{8}$ years with the Mercury package described in Chambers (1999). Mercury is a hybrid integrator, made of a conventional Burlish-Stoer integrator and a symplectic integrator. The symplectic integrator is preferred since it exhibits no long-term accumulation of energy error, and is fast. A timestep of 2 days was adopted since the inner body is at $0.4 \mathrm{AU}$ from the star.

We performed some tests to verify the reliability of the symplectic algorithm in the presence of a massive perturber as Alpha Centauri B. Massless particles have been integrated for $10 \mathrm{Myr}$ with semimajor axes of 2.0, 2.25, and 2.5, and eccentricity of $0,0.2,0.5$ and 0.7 with both the symplectic and 
Table 1. Physical properties of the stars in $\alpha$ Centauri system. (a) Pourbaix et al. (1999); (b) Benedict et al. (1999).

\begin{tabular}{cccc}
\hline \hline star & $\begin{array}{c}\text { mass } \\
M_{\odot}\end{array}$ & MK class & ref. \\
\hline A & 1.1 & G2 V & a \\
B & 0.92 & K0 V & a \\
C & 0.11 & M5 V & b \\
\hline
\end{tabular}

the Radau (Everarth 1985) integrators. The region between 2.0 and $2.5 \mathrm{AU}$ is the outermost zone initially populated by protoplanets in our simulations and the most critical since the perturbations of the companion star are stronger. The outcome of the two algorithms were in good agreement and significative differences were found only for highly eccentric orbits $(e=0.5,0.7)$ at $2.5 \mathrm{AU}$ : the bodies escape on hyperbolic orbits in both the simulations, but at different times depending on the integrator used. However, this discrepancy is not due to a bad approximation in the symplectic algorithm but to the chaotic behaviour of eccentric bodies close to the 2.5 AU location. To confirm this supposition, we integrated with Radau two clones of the particles started at 2.5 AU and with eccentricity of 0.5 and 0.7 , respectively. The two clones were obtained by changing the 9th digit of the semimajor axis of the original test particles. We found again a significant change (in one case of an order of magnitude) in the escaping time even if the same integrator was used (Radau). As a further test we verified that the relative energy change in our simulations was of the order of $10^{-8}$. The Hamiltonian decomposition at the base of the symplectic algorithm is then still effective, at least within 2.5 AU from the star. Additional tests have also been performed in the inner region of the protoplanet disk. The numerical simulations with the two integrators showed no discrepancy, even for large values of eccentricity, for a semimajor axis $a=0.5 \mathrm{AU}$, the inner value used in our starting conditions. The choice of a short timestep ( 2 days) for the symplectic algorithm was critical in this respect.

Collisions between embryos are assumed to be completely inelastic; fragmentation or cratering are not considered. This is a good approximation since the gravitational binding energy for large bodies overcomes the kinetic energy of the fragments also at high velocity impacts. A single body is left after a collision and its orbit is determined by the conservation of linear momentum.

The integrations were simultaneously performed in a local CONDOR pool (http://www.cs. wisc . edu/ condor) made of about 200 Pentium class computers with an average speed of 200 Mflops. A single simulation needed about 2 months of real time.

\section{Initial conditions}

$\alpha$ Centauri is a triple system with two of the stars forming a close binary (HD 128620 and HD 128621) and a third (GJ 551) orbiting this pair at a much greater distance. The physical properties of the stars in the system are summarized in Table 1.

In our model, we ignore the distant third star, $\alpha$ Cen $\mathrm{C}$ (Proxima), as it might not be bound to the central binary system
(Anosova et al. 1994). Moreover, its perturbations on the embryo orbits would be small. The star $\alpha$ Cen B moves on a fixed elliptical orbit around the primary component A, with a semimajor axis of 23.4 AU and an eccentricity of 0.52 (Soderhjelm 1999). The protoplanets move around component A, and they are set on the orbital plane of the binary system with a low inclination with respect to it. This assumption is based on the work of Hale (1994). He shows that if the separation between the components of a binary system is around 30-40 AU or less, the components of observed binary systems are coplanar with respect to their mutual orbit and their equator.

To compute the initial distribution of embryos around the primary star, we have adopted a surface density of solid material $\sigma$ equal to $8 \mathrm{~g} \mathrm{~cm}^{-2}$ at $1 \mathrm{AU}$, the same value adopted by Chambers (2001) and intermediate between that used by Kokubo \& Ida (1996) and that adopted by Chambers \& Wheterill (1997) for the solar nebula. Since we cover a large range in semimajor axis of the initial embryos, we assume that the surface density decreases as $r^{-3 / 2}$ (Weidenschilling et al. 1997). Of course these are tentative assumptions, since no data are available. Observations of circumstellar disks in the binary system L1551 by Rodriguez et al. (1998) seem to suggest high density values: for a disk extending only for about 10 AU they derive masses of the order of 3 times the minimum mass of the solar nebula. However, in this case the evolutive state of the accretion disk and whether material is still falling onto the star is not known.

In the simulations we used different values for the embryo's initial mass ranging from $0.75 M_{\mathrm{L}}\left(\sim 0.01 M_{\oplus}\right)$ to $5.00 M_{\mathrm{L}}$ $\left(\sim 0.06 M_{\oplus}\right)$. The total mass in the embryos was about $75 \%$ of that expected in a disk with $\sigma=8 \mathrm{~g} \mathrm{~cm}^{-2}$ since a consistent fraction possibly still resides in small planetesimals. Depending on the initial borders of the disk of planetary embryos, their total mass ranged from 1.47 to $2.61 M_{\oplus}$. The maximum outer edge considered for the disk was 2.79 , AU in agreement with the limit for orbital stability of Holman \& Wiegert (1999), while the inner border was set to $0.4 \mathrm{AU}$. The protoplanets radii, are calculated assuming a density of $3 \mathrm{~g} \mathrm{~cm}^{-3}$ typical for planetary embryos in the terrestrial planet region (Chambers \& Wheterill 1997). The initial eccentricities are randomly selected in the range $0 \leq e \leq 0.04$, the inclinations in the range $0^{\circ} \leq i \leq 0.6^{\circ}$. The remaining orbital elements are chosen randomly between $0^{\circ}$ and $360^{\circ}$. The initial separation between the planetary embryos, $\Delta$, is measured in mutual Hill radii, defined by the equation:

$R_{\mathrm{H}}=\left(\frac{m_{i}+m_{i+1}}{3 M_{\alpha \mathrm{CenA}}}\right)^{1 / 3}\left(\frac{a_{i}+a_{i+1}}{2}\right)$

where $i$ refers to the $i$ th body, specifically to the mass and to the orbital semimajor axis, $M_{\alpha}$ CenA is the mass of $\alpha$ Cen A. We varied the value of $\Delta$ from $\sim 3$ to 9 in the different simulations to test how this parameter influences the outcome.

The initial parameters are collected in Table 2. 
Table 2. List of the initial parameters for each of the 26 simulations. The parameters are: $n$, the number of bodies in each simulation; $a_{1}$, the orbital semimajor axis of the inner body in $\mathrm{AU} ; a_{n}$, the orbital semimajor axis of the outer body in $\mathrm{AU} ; m_{i}$, the mass of each body in Moon masses (Moon mass $=7.35 \times 10^{22} \mathrm{~g}$ ); $m_{\mathrm{T}}$, the total mass of the disk of protoplanetary embryos in Earth masses (Earth mass = $5.976 \times 10^{24} \mathrm{~g}$ ); and $\Delta$, the separation between the bodies in mutual Hill radii.

\begin{tabular}{cccccc}
\hline \hline$n$ & $a_{1}$ & $a_{n}$ & $m_{i}$ & $m_{\mathrm{T}}$ & $\Delta$ \\
& $\mathrm{AU}$ & $\mathrm{AU}$ & $M_{\mathrm{moon}}$ & $M_{\oplus}$ & $R_{\mathrm{H}}$ \\
\hline & & & & & \\
100 & 0.55 & 1.64 & 1.25 & 1.53 & 3.65 \\
100 & 0.55 & 1.93 & 1.50 & 1.83 & 3.95 \\
100 & 0.55 & 2.24 & 1.75 & 2.14 & 4.19 \\
100 & 0.55 & 2.58 & 2.00 & 2.44 & 3.11 \\
77 & 0.75 & 2.10 & 1.75 & 1.65 & 4.00 \\
64 & 0.55 & 1.68 & 2.00 & 1.56 & 4.00 \\
128 & 0.50 & 1.82 & 1.10 & 2.02 & 3.50 \\
175 & 0.50 & 2.37 & 1.05 & 2.57 & 3.13 \\
112 & 0.50 & 1.74 & 1.20 & 1.94 & 3.75 \\
36 & 0.50 & 2.36 & 5.00 & 2.57 & 9.00 \\
39 & 0.50 & 2.27 & 4.50 & 2.47 & 8.50 \\
41 & 0.50 & 2.14 & 4.00 & 2.35 & 8.00 \\
44 & 0.50 & 1.98 & 3.50 & 2.18 & 7.50 \\
45 & 0.50 & 2.10 & 3.50 & 2.10 & 7.00 \\
53 & 0.50 & 1.75 & 3.00 & 1.94 & 7.00 \\
52 & 0.50 & 1.92 & 3.00 & 1.91 & 6.50 \\
52 & 0.50 & 1.61 & 2.50 & 1.59 & 6.00 \\
82 & 0.50 & 2.11 & 2.00 & 2.32 & 5.00 \\
150 & 0.55 & 1.93 & 1.00 & 1.83 & 3.00 \\
95 & 0.55 & 1.84 & 1.50 & 1.74 & 4.00 \\
117 & 0.55 & 2.65 & 1.75 & 2.50 & 4.00 \\
137 & 0.75 & 2.58 & 1.25 & 2.01 & 3.00 \\
149 & 0.50 & 2.43 & 1.25 & 2.61 & 3.50 \\
131 & 0.50 & 1.33 & 0.75 & 1.47 & 2.95 \\
77 & 0.75 & 2.10 & 1.75 & 1.65 & 4.00 \\
64 & 0.55 & 1.68 & 2.00 & 1.56 & 4.00 \\
\hline & & & & &
\end{tabular}

\section{Typical evolution of a population of planetary embryos into planets}

Here we present the evolution of a typical simulation of planetary formation in $\alpha$ Centauri taken from our set of 26 numerical cases. The initial parameters are given in Table 2 (simulation number 2, hereinafter SIM2). In Fig. 1 we show the time evolution of the semimajor axis versus eccentricity for all the bodies at different evolutionary times. Already after $10^{5}$ years the orbits of planetary embryos are dynamically excited with eccentricities up to 0.1. At subsequent times the eccentricities grow even further for the combination of the companion star perturbations and mutual encounters and collisions. After $1 \times 10^{8}$ years three planetary bodies are left: the two largest planets have a mass similar to the mass of Venus while the third one has two times the mass of Mars. The final orbital parameters are given in Table 3.

In Fig. 2 we illustrate the time evolution of the semimajor axes of each body in the simulation. After a brief chaotic evolution when all the bodies undergo repeated mutual close encounters, the number of bodies begins to decrease with time because
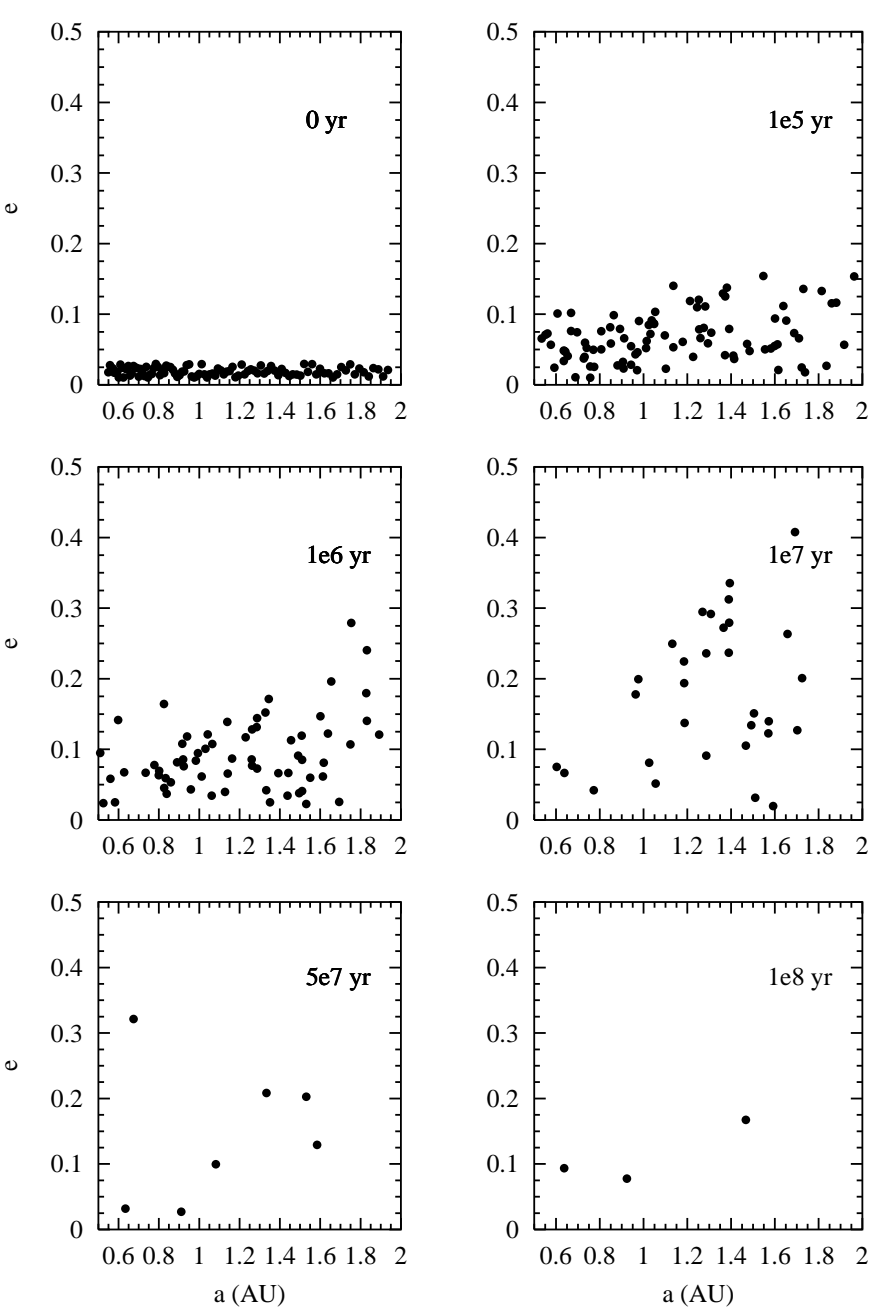

Fig. 1. Snapshots at various times of the orbital semimajor axis $a$ versus the eccentricity $e$ for all the bodies in SIM2.

Table 3. Orbital elements for the surviving planets in SIM2.

\begin{tabular}{rrrrrrrr}
\hline \hline & $\begin{array}{r}\text { mass } \\
M_{\oplus}\end{array}$ & $\begin{array}{r}a \\
\mathrm{AU}\end{array}$ & $e$ & $\begin{array}{r}i \\
{ }^{2}\end{array}$ & $\begin{array}{r}\omega \\
{ }^{\circ}\end{array}$ & $\begin{array}{r}M \\
{ }^{\circ}\end{array}$ & $\begin{array}{r}N \\
{ }^{\circ}\end{array}$ \\
\hline B02 & 0.550 & 0.637 & 0.094 & 1.7 & 209 & 115 & 333 \\
B31 & 0.715 & 0.924 & 0.078 & 1.5 & 35 & 186 & 218 \\
B36 & 0.202 & 1.467 & 0.167 & 3.4 & 327 & 67 & 325 \\
\hline
\end{tabular}

of mutual collisions and consequent accumulation into larger bodies. Some of the initial embryos also impact on each of the two stars or are ejected out of the system. After $5 \times 10^{7}$ years most of the bodies have accreted into larger ones and only a few bodies are involved in the evolution of the system in the subsequent $5 \times 10^{7}$ years. The three surviving planets at the end of the simulation are in non-crossing orbits and form an apparently stable system.

The SIM2 is peculiar for its final dynamical configuration: the outer planet and the intermediate one are locked in a $2: 1$ mean motion resonance. This confirms that resonant configurations may be a common outcome of the planetary formation process. Even two observed exoplanetary systems have their planets possibly locked in a 2:1 resonance, i.e. GJ 876 


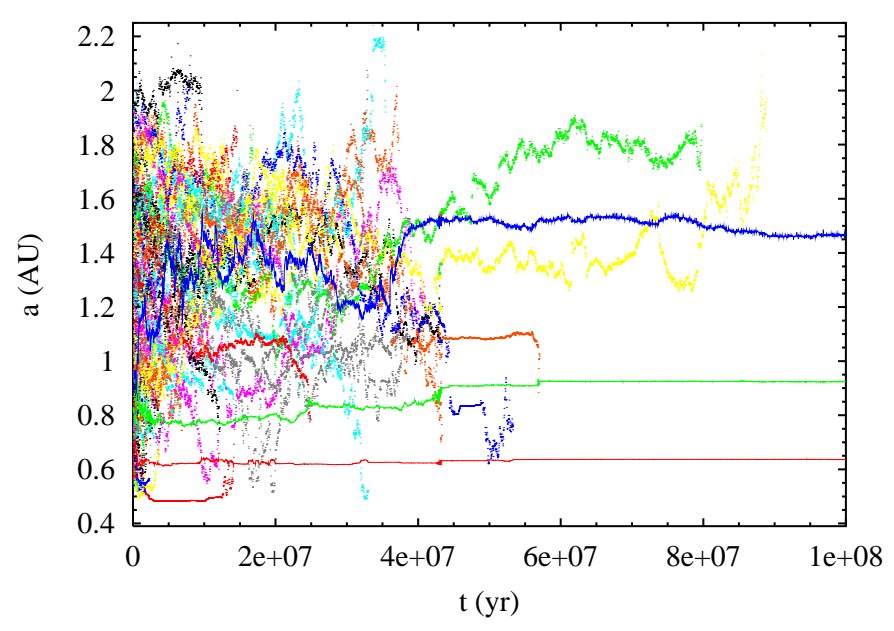

Fig. 2. Orbital semimajor axis as function of time for all the bodies in simulation 2 . The surviving bodies are represented by continuous lines.

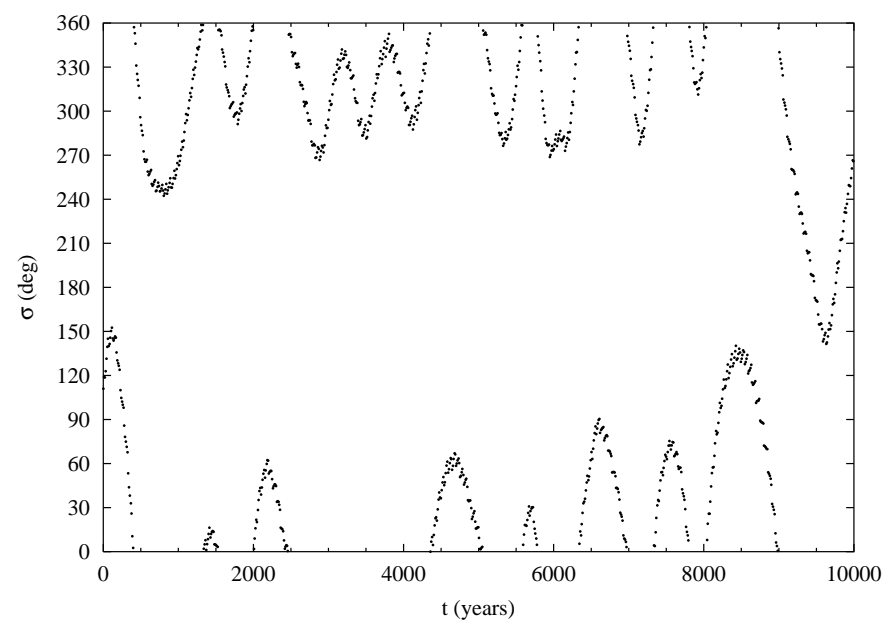

Fig. 3. Critical argument $\sigma=2 \lambda_{2}-\lambda_{1}-\tilde{\omega}_{1}$ of the $2: 1$ mean motion resonance detected between two planets at the end of SIM2. $\lambda_{1}$ and $\tilde{\omega}_{1}$ are the mean longitude and perihelion longitude of the inner planet whose mass is $0.72 M_{\oplus}$ and semimajor axis $0.92 \mathrm{AU} ; \lambda_{2}$ is the mean longitude of the outer planet whose mass is $0.2 M_{\oplus}$ and semimajor axis $1.46 \mathrm{AU}$.

(Marcy et al. 2001) and HD 82943 (Goździewski \& Maciejewski 2001).

In Fig. 3 we show the critical argument $\sigma=2 \lambda_{2}-\lambda_{1}-\tilde{\omega}_{1}$ of the $2: 1$ resonance over a period of $1 \times 10^{4}$ years, where $\lambda_{1,2}$ are the mean longitudes of the inner and outer planets respectively and $\tilde{\omega}_{1}$ is the longitude of periapse of the inner planet. The resonant state between the two planets is however unstable and after $2.3 \times 10^{8}$ years the outer planet begins to evolve chaotically, its eccentricity grows, and it is ejected out of the system leaving only the two major planets in orbit around $\alpha$ Cen A.

\section{Statistics for all the simulations}

In this section we combine the outcome of all the 26 simulations to produce statistical predictions on the planetary formation process in $\alpha$ Centauri. In Fig. 4 we plot the final mass of the planets that formed in all the simulations as a function of their

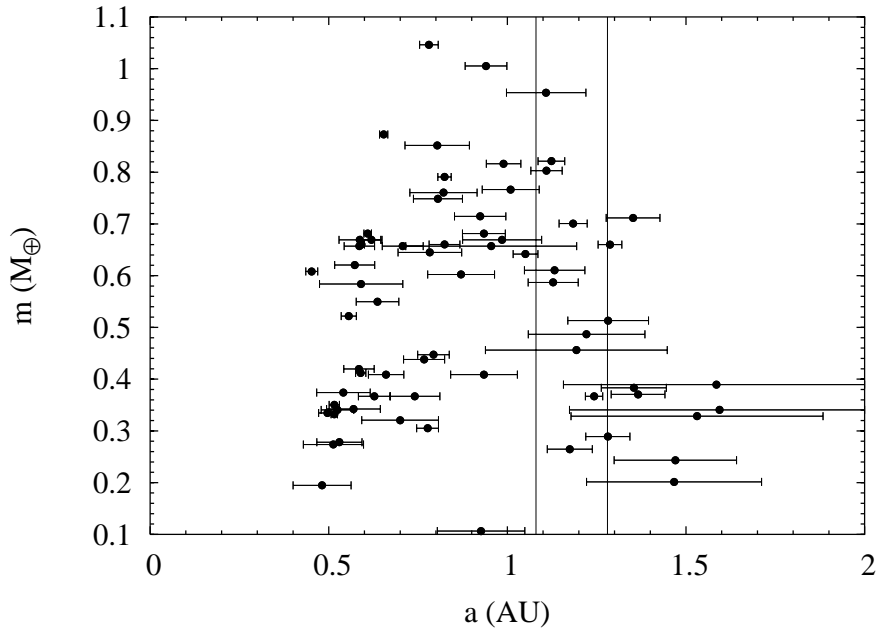

Fig. 4. Distribution of semimajor axis vs. mass of all the terrestrial planets that formed in our simulations. The vertical lines are the edges of the habitable zone for $\alpha$ Cen A. The horizontal bars show the perihelion-aphelion distances.

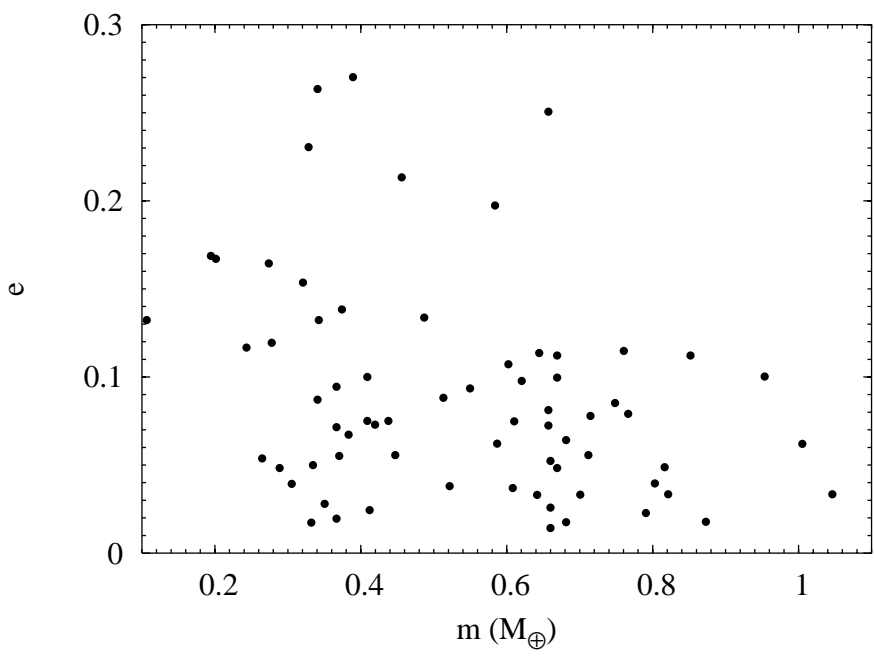

Fig. 5. Eccentricity versus mass for all the planets formed at the end of our simulations.

semimajor axis. The horizontal bars define the perihelion and aphelion distance for each body. The mass values range from 0.2 to $\sim 1 M_{\oplus}$ and some of them have their orbits within the habitable zone for $\alpha$ Cen A that, in the plot, is limited by two vertical bars. The maximum semimajor axis is $\sim 1.6 \mathrm{AU}$ and bodies closer to this outer limit have larger values of eccentricity due to the gravitational perturbations of the companion star. The eccentricity vs. mass distribution shown in Fig. 5 confirms that some planets, in particular the inner ones, end up in almost circular orbits.

Our Figs. 4 and 5 may be compared with Fig. 11 and 12 of Chambers (2001) that illustrate similar results concerning the accretion of terrestrial planets in the solar system in presence of both Jupiter and Saturn as perturbing planets. Since we used in our simulations the same value of $\sigma$ and similar values for the initial mass and spacing of the embryos have been used, the comparison gives an insight on the expected differences between the planetary formation process in the two systems. 


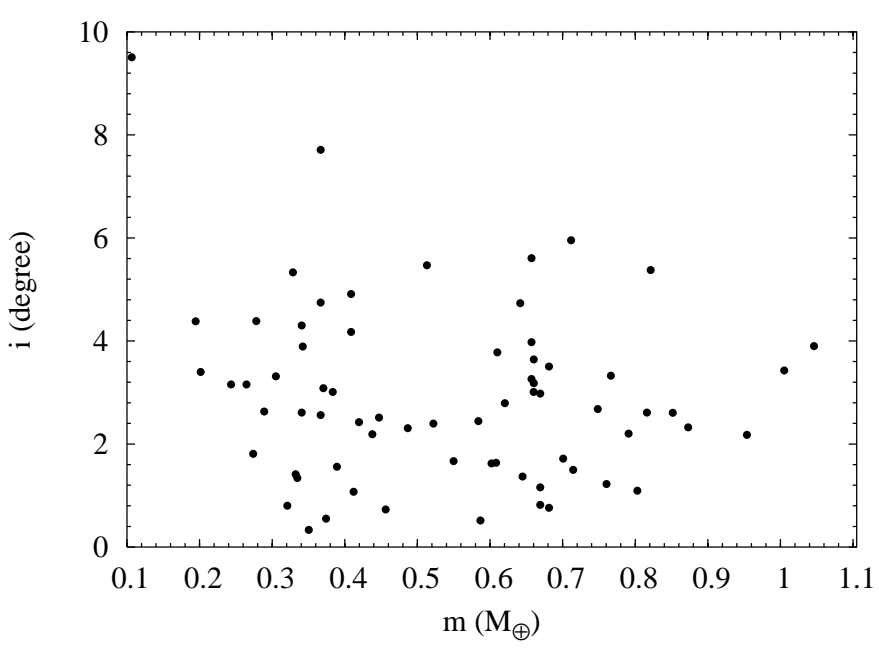

Fig. 6. Mass of the final planets versus their orbital inclination.

A similar triangular pattern is observed in the distribution of the final planetary mass vs. semimajor axis (our Figs. 4 and 11 of Chambers 2001) but more massive planets may form in the inner solar system, up to $1.5 M_{\oplus}$, compared to $\alpha$ Cen where the maximum mass value is $1 M_{\oplus}$. In the simulations of Chambers (2001) low mass terrestrial planets may accumulate also beyond 1.5 AU, up to $2 \mathrm{AU}$ while for $\alpha$ Cen we do not get planets beyond 1.6 AU. In both systems the eccentricities are on average lower than 0.15 but we find a higher percentage of low eccentricity orbits in particular for the inner planets. Moreover, in our Fig. 5 there is a more clear trend towards higher values of eccentricity for less massive bodies.

A possible planetary system around $\alpha$ Cen A would then be very similar to the inner solar system with two or three terrestrial planets with size ranging from a Mars-like planet to an Earth-like planet. This is a somewhat surprising result taking into account the strong perturbations of the companion star.

The distribution of the final inclinations is shown in Fig. 6 as a function of the final mass of the planets. High inclinations, of the order of some degrees, may be achieved in particular by the smaller bodies. For the larger planets the inclination is comparable to that of the terrestrial planets in our Solar System.

To gain more insight on the evolution of planetary embryos into planets, we have plotted in Fig. 7 the mass growth as a function of time of all the planets shown in Fig. 4. For each body the mass value is normalized to the final value. The majority of the virtual planets gain most of their mass within $4.0 \times 10^{7}$ years while in the subsequent years only about $10 \%$ of the final mass is accreted. We can then set to $4.0 \times 10^{7}$ years the time-scale within which most of the accretion occurs.

In Fig. 8 we show the final values of the obliquity of the planets. The distribution is random with a peak around $80^{\circ}$. Only a small fraction has an obliquity close to $0^{\circ}$ that represents the state of our Solar System with most of the planets having the spin axes perpendicular to the orbital plane. Similar results on the obliquity were also obtained by Chambers (2001). One possible explanation is that in our simulations we have not considered the effects of fragmentation and hence the possibility of formation of satellites that might reduce the obliquity (as in the case of Earth and Moon).

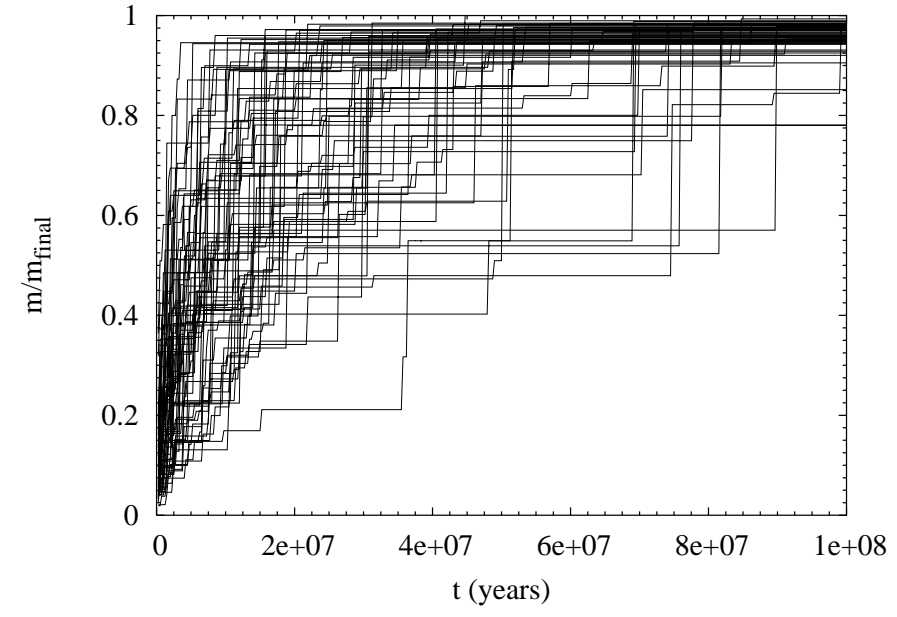

Fig. 7. Mass growth of the embryos that become planets vs. time. The value of mass of each body is normalized to its final mass as a planet.

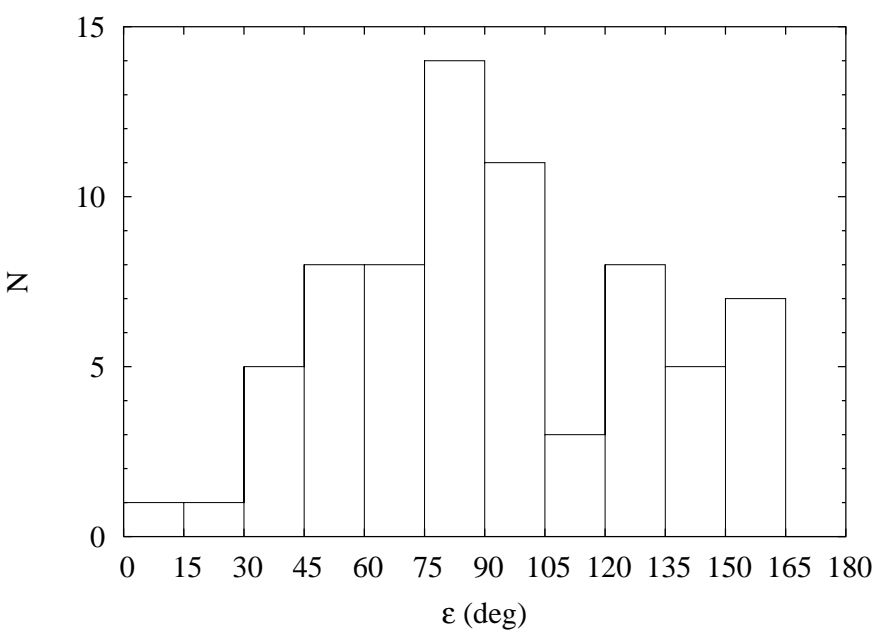

Fig. 8. Histogram of the obliquities of the final planets.

At the end of each integration we have checked whether the final planets are in some kind of mean motion resonance. In 6 simulations out of 26, two of the final planets were close within $1 \%$ in semimajor axis to a low order mean motion resonance $(2: 1$ or $3: 2)$. In one case, that described in Sect. 4 and termed SIM2, the two planets were in resonance as tested by the libration of the critical argument. This suggests that resonant configurations in planetary systems may not be rare. To give statistical robustness to this statement we should however estimate the width of the main resonances (possibly as a function of the eccentricity) and compare it with the semimajor axis range available for stable orbital configurations of the planets. We could then state that 6 cases out of 26 is a statistical significant result and not just a random outcome. Such an investigation is however out of the scope of this paper.

\section{Ingestion of planetary material by the parent stars}

During the process of planetary formation some embryos, because of the strong perturbations of the binary companion, impact on the primary or on the secondary star. In all our 


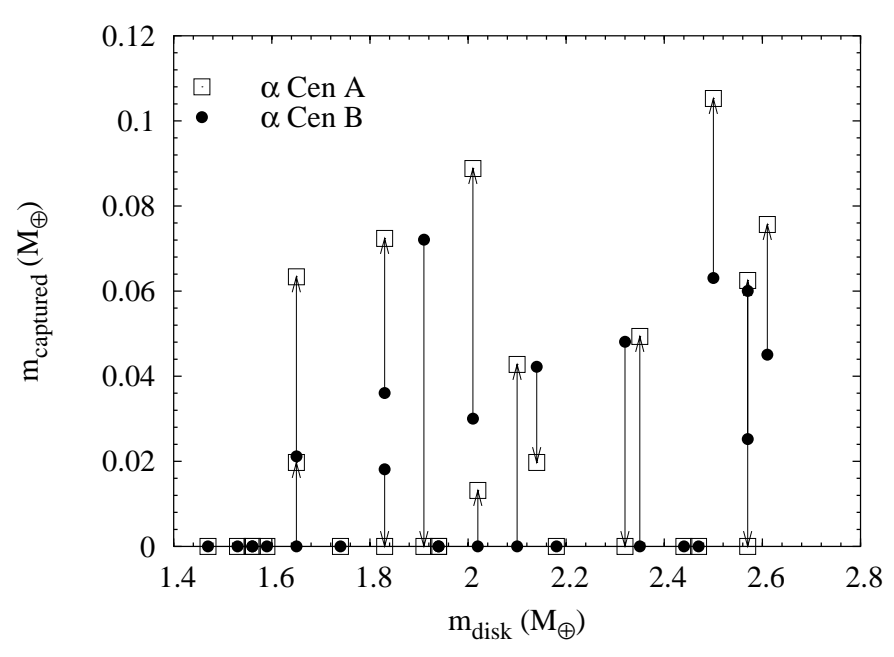

Fig. 9. Amount of mass captured by the two stars during the growth of terrestrial planets around $\alpha$ Cen A.

simulations we compute the fraction of the initial mass that ends up in either of the stars. In Fig. 9 we show the amount of mass plunging into $\alpha$ Cen $\mathrm{A}$ and $\mathrm{B}$, respectively. On average, about $2 \%$ of an Earth mass is engulfed by either of the stars, so the metallicity of the stars should not have been significantly altered in this final stage of the planetary accretion process. At least $0.5 M_{\oplus}$, according to Murray et al. (2001), are needed to alter noticeably the metallicity of a main-sequence star. However, we started our simulations with a protoplanetary disk whose density was equal to that expected in the primordial solar nebula. More massive initial disks would lead to significantly higher amount of mass falling onto the two stars, possibly affecting the composition of the convective envelope. From Fig. 9 we also notice that there may be an asymmetry between the amount of material plunging onto the two stars and, as a consequence, there might be a difference in the metallicity of a close binary system if planetary accretion occurred only around one of the components.

We have to point out that the previous results on the mass infall of material on the stars must be taken as indicative in a statistical sense. Numerical integrations of highly eccentric protoplanetary trajectories may be not very accurate when the bodies are far from the primary body. When a protoplanet in a high eccentric orbit travels around the aphelion and then closer to the companion star, the orbital integration may accumulate a significant integration error. However, being the orbits easily chaotic, the outcomes of our integration in this case may be taken as predictive only in terms of probability of impact onto the two stars among the escaping bodies.

\section{Conclusions}

Planetary formation seems to be possible in the Alpha Centauri system and, in general, in close binary systems. Marzari \& Scholl (2000) showed that planetesimals can accrete into planetary embryos; in this paper we demonstrate that planetary embryos can grow into planets in about $50 \mathrm{Myr}$. These terrestrial-size planets form within the region of planetary stability found by Wiegert \& Holman (1997) and are possibly stable over the age of the system.

Assuming that the initial mass in the protoplanetary disk around the main component of the $\alpha$ Cen system was comparable to that of the minimum solar nebula, planets with the size of Mars and Venus are typically formed with low orbital eccentricity. Some of them lay within the habitable zone of the star and, possibly, could harbor life.

During the accumulation process, some of the planetary embryos are engulfed by the stars in different amounts. However, only if the protoplanetary disk was very massive, at least five times more than the minimum mass solar nebula, we might observe a difference in the metallicity of the star related to the ingestion process.

Orbital resonances may occur between the planets. In a significant fraction of our numerical simulations we found an orbital period close to a low order commensurability ratio. In one case we also found a real 2:1 resonance between two planets, with the critical argument librating around 0 . This system was stable over $200 \mathrm{Myr}$ after which the outer planet entered a chaotic state and was ejected out of the system.

Acknowledgements. We thank the referee J. J. Lissauer for useful suggestions.

\section{References}

Anosova, J., Orlov, V. V., \& Pavlova, N. A. 1994, A\&A, 292, 115

Benedict, G. F., McArthur, B., Chappell, D. W., et al. 1999, AJ, 118, 1086

Chambers, J. G., \& Wheterill, G. 1998, Icarus, 136, 304

Chambers, J. E. 1999, MNRAS, 304, 793

Chambers, J. E. 2001, Icarus, 152, 205

Everhart, E. 1985, in Dynamics of comets: their origin and evolution, ed. A. Carusi, \& G. B. Valsecchi (Reidel, Dordrecht), 185

Goździewski, K., \& Maciejewski, A. J. 2001, ApJ, 563, L81

Hale, A. 1994, AJ, 107, 306

Holman, M. J., \& Wiegert, P. A. 1999, AJ, 117, 621

Kokubo, E., \& Ida, S. 1996, Icarus, 123, 180

Marcy, G. W., Butler, R. P., Fischer, D., et al. 2001, ApJ, 556, 296

Marzari, F., \& Scholl, H. 2000, ApJ, 543, 328

Murray, N., Chaboyer, B., Arras, P., Hansen, \& Noyes, R. W. 2001, ApJ, 555, 801

Pourbaix, D., Neuforge-Verheecke, C., \& Noels, A. 1999, A\&A, 344, 172

Rodriguez, L. F., D’Alessio, P., Wilner, D. J., et al. 1998, Nature, 395, 355

Soderhjelm, S. 1999, A\&A, 341, 121

Weidenschilling, S. J., Spaute, D., Davis, D. R., Marzari, F., \& Ohtsuki, K. 1997, ICARUS, 128, 429

Wiegert, P. A., \& Holman, M. J. 1997, AJ, 113, 1445 